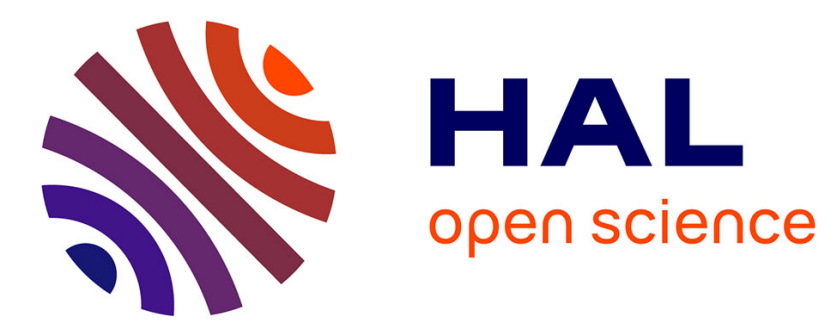

\title{
Geostrophic adjustment on the equatorial beta-plane revisited
}

\author{
Masoud Rostami, V. Zeitlin
}

\section{To cite this version:}

Masoud Rostami, V. Zeitlin. Geostrophic adjustment on the equatorial beta-plane revisited. Physics of Fluids, 2019, 31 (8), pp.081702. 10.1063/1.5110441 . hal-02266066

\section{HAL Id: hal-02266066 \\ https://hal.science/hal-02266066}

Submitted on 13 Aug 2019

HAL is a multi-disciplinary open access archive for the deposit and dissemination of scientific research documents, whether they are published or not. The documents may come from teaching and research institutions in France or abroad, or from public or private research centers.
L'archive ouverte pluridisciplinaire HAL, est destinée au dépôt et à la diffusion de documents scientifiques de niveau recherche, publiés ou non, émanant des établissements d'enseignement et de recherche français ou étrangers, des laboratoires publics ou privés. 


\section{Geostrophic adjustment on the equatorial beta-plane revisited}

Cite as: Phys. Fluids 31, 081702 (2019); https://doi.org/10.1063/1.5110441

Submitted: 17 May 2019 . Accepted: 27 July 2019 . Published Online: 12 August 2019

M. Rostami, and V. Zeitlin

$\frac{4}{6}$

\section{CAPTURE WHAT'S POSSIBLE}

WITH OUR NEW PUBLISHING ACADEMY RESOURCES

Learn more $\Theta$

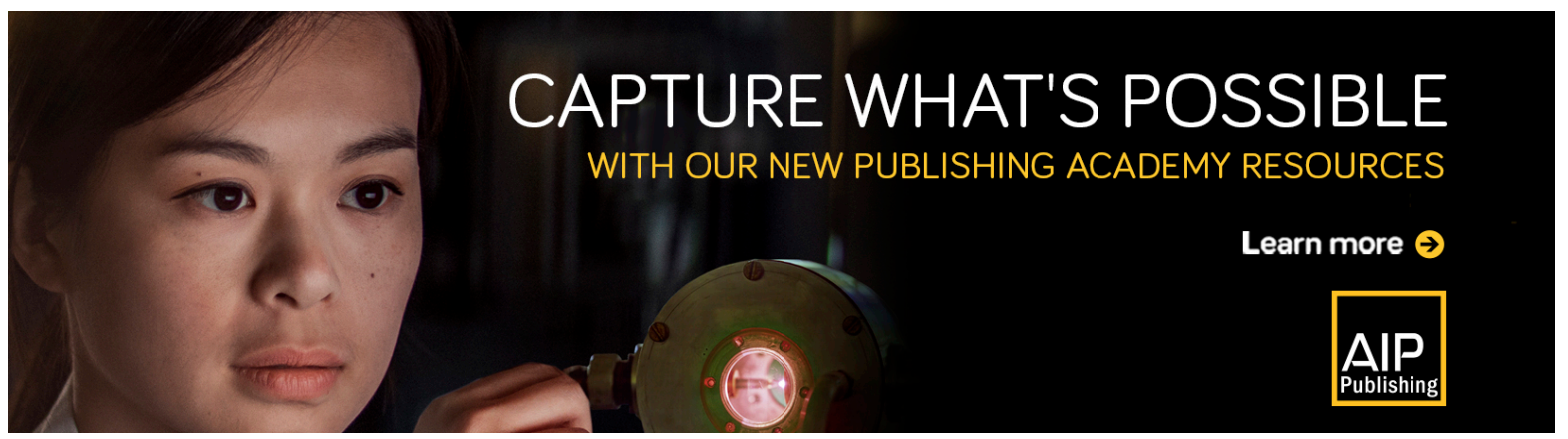


Geostrophic adjustment on the equatorial beta-plane revisited

3

4 Su

5

6

7

8

9

10

11

\section{MASOUD ROSTAMI $^{a, b}, \operatorname{VLADIMIR~ZEITLIN~}^{a}$}

${ }^{a}$ Laboratoire de Météorologie Dynamique (LMD)/IPSL, Sorbonne Universités, Ecole Normale Supérieure(ENS), Paris, France, and Department of Mathematics, Southern University of Science and Technology, Shenzhen, 518055, China

${ }^{b}$ Institute for Geophysics and Meteorology (IGM), University of Cologne, Cologne, Germany

This is the Authors Original Manuscript (AOM); that is, the manuscript in its original form; a preprint . The Version of Record of this manuscript has been published and is available in the: “AIP Publishing - Physics of Fluid": https://doi .org/10.1063/1.5110441 


\title{
Geostrophic Adjustment on the equatorial beta-plane revisited
}

\author{
M. Rostami ${ }^{1,2}$, V. Zeitlin ${ }^{1 *}$ \\ ${ }^{1}$ LMD, Sorbonne University and Ecole Normale Supérieure, \\ 24 rue Lhomond, 75005 Paris, France, and Department of Mathematics, \\ Southern University of Science and Technology, Shenzhen, 518055, China \\ ${ }^{2}$ Institute for Geophysics and Meteorology (IGM), \\ University of Cologne, Cologne, Germany
}

\begin{abstract}
The process of geostrophic adjustment of localized large-scale pressure anomalies in the standard adiabatic shallow-water model on the equatorial beta-plane is revisited, and it is shown that the standard scenario of generation of westward-moving Rossby and eastward-moving Kelvin waves, which underlies the classical Gill theory of tropical circulation due to a localized heating, is not unique. Depending on strength and aspect ratio of the initial perturbation, the response to the initial perturbation in the western sector can be dominated by inertia-gravity waves. The adjustment in the diabatic moist-convective shallow water model can be totally different, and produces, depending on parameters, either Gill-like response, or eastwardmoving coherent dipolar structures of the type of equatorial modons, which do not appear in the "dry" adjustment, or vortices traveling, respectively, North-West in the Northern, and South-West, in the Southern hemispheres.
\end{abstract}

\footnotetext{
* Corresponding author. Email: zeitlin@lmd.ens.fr
} 
The famous Gill theory of the response of the tropical atmosphere to localised large-scale heating [1], which consists in generation of Rossby wave like and Kelvin wave like responses at the western and eastern sides of the source, respectively, is widely known. It has become a folklore in tropical meteorology and climatology, and is ubiquitously evoked in the literature for explanation of observations and numerical simulations. The Gill mechanism is intrinsically related to the process of geostrophic adjustment in tropics, i.e. relaxation of large-scale perturbations by emission of equatorial waves, because the heating source, which was represented in original papers $[1,2]$ and subsequent studies, e.g. [3], as a mass-sink in the shallow-water model of the atmosphere, can be considered as permanently regenerating a localised negative pressure anomaly. The notion of geostrophic adjustment, borrowed from the theory of relaxation of large-scale perturbations in mid-latitudes, e.g. [4], has a specific meaning in the tropics as, due to the absence of the constant part of the Coriolis parameter at the Equator, the geostrophic balance is well-defined only for the zonal velocity. Yet, the equatorial Rossby waves are approximately geostrophic, as was noticed already in the pioneering paper by Matsuno [5], and the geostrophic ansatz for both components of velocity allows to recover Rossby waves from the linearized potential vorticity equation [6]. The zonal velocity of the equatorial Kelvin waves is in geostrophic equilibrium, as well. Such equilibrium arises naturally for equatorial motions with a strong disparity between meridional (smaller) and zonal (larger) scales, [7], Sect. 5.5.4. This scaling was implicitly used in [1], by imposing the equatorial geostrophic balance. Non-linear theory of equatorial geostrophic adjustment under this scaling was developed inshallow-water model on the equatorial beta-plane, and confirmed by numerical simulations in [8], with the results fully consistent with the Gill mechanism.

Yet, the large-scale perturbations with typical scales of the order of the equatorial deformation radius, could have comparable zonal and meridional scales, and a question arises how the scenario of geostrophic adjustment changes in this case. This question, as to our knowledge, was not addressed in the literature, except for the paper by Matsuda and Takayama [9], where numerical simulations of the adjustment on the sphere revealed different scenarios in the equatorial region, depending on the parameters of initial perturbations. The dominant heating source in the equatorial atmosphere is the moist convection. Therefore, another question arises on changes of the equatorial adjustment in a moist-convective environment, when the evolution of moisture and moist convection, with related latent heat release, are incorporated in the model. The answers to these questions are given below. We show, first, that the "dry" geostrophic adjustment is strongly sensitive to the aspect ratio of the initial perturbations, and can be dominated by large-scale westward- 
propagating inertia-gravity waves, instead of Rossby waves in the classical scenario. Second, we show that the relaxation of pressure anomalies in the moist-convective environment may follow the Gill scenario, but may also produce eastward- moving cyclonic dipoles, and vortices detaching from the Equator, which does not happen in the "dry" case.

Rotating shallow water (RSW) on the equatorial beta-plane is the archetype model for atmospheric dynamics in tropics $[1,5]$. We are using this model, which will be understood as vertically averaged primitive equations in pseudo-height pressure coordinates [7]. The model can be extended to include water vapor with condensation and related latent heat release [10], as well as surface evaporation. Liquid water, precipitation, vaporization and related cooling can be also incorporated in the model, as well as vertical structure, see [11] and references therein, but below we will be using the simplest one-layer version of such moist-convective RSW (mcRSW).

The equations of the "dry" adiabatic one-layer RSW model in the equatorial beta- plane with no topography, and no dissipation read:

$$
\left\{\begin{array}{l}
\partial_{t} \mathbf{v}+\mathbf{v} \cdot \nabla \mathbf{v}+\beta y \hat{\mathbf{z}} \wedge \mathbf{v}+g \nabla h=0, \\
\partial_{t} h+\nabla \cdot(\mathbf{v} h)=0
\end{array}\right.
$$

where $\nabla=\left(\partial_{x}, \partial_{y}\right), \mathbf{v}=(u, v), u$ is zonal and $v$-meridional components of velocity, $h$ is geopotential height (thickness), $\beta$ is the meridional gradient of the Coriolis parameter, and $\hat{\mathbf{z}}$ is a unit vertical vector. In the simplest version the equations of the diabatic mcRSW [10] read:

$$
\left\{\begin{array}{l}
\partial_{t} \mathbf{v}+\mathbf{v} \cdot \nabla \mathbf{v}+\beta y \hat{\mathbf{z}} \wedge \mathbf{v}=-g \nabla h, \\
\partial_{t} h+\nabla \cdot(\mathbf{v} h)=-\gamma P, \\
\partial_{t} Q+\nabla \cdot(Q \mathbf{v})=-P+E .
\end{array}\right.
$$

Here $Q$ is a bulk amount of water vapor in the air column, $\gamma$ is a parameter depending on the underlying stratification, $P$ is the condensation sink, and $E$ is the evaporation source of moisture, which are parameterised as follows:

$$
P=\frac{Q-Q^{s}}{\tau} \mathscr{H}\left(Q-Q^{s}\right), \quad E=\alpha|\mathbf{v}|\left(Q^{s}-Q\right) \mathscr{H}\left(Q^{s}-Q\right) .
$$

$Q^{s}$ is a saturation moisture threshold, $\tau$ is relaxation time, $\alpha$ is a parameter regulating evaporation, and $\mathscr{H}(\ldots)$ denotes the Heaviside (step-)function.

Notice that, similar to the Gill's theory, convective heating acts as a forcing in the mass conservation equation, although here it is not $a d h o c$, but is determined by the evolution of moisture, 
which, in turn, is advected by the velocity field, and is conserved in the air column, unless the condensation and evaporation are switched on.

Below we present the results of numerical simulations, first of the "dry", and then of the moistconvective (MC) RSW equations, initialized with localized large-scale pressure anomalies $h=$ $H_{i}(x, y)$ of the so-called alpha-Gaussian shape [12], symmetric with respect to the equator. The circular anomaly $H_{i}(r)$ normalized by the mean thickness is given by the formula $H_{i}(r)=1+$ $\varepsilon \frac{2^{1 / s}}{s} \Gamma\left(\frac{1}{s}+\frac{1}{2}\right) G\left(\frac{r^{s}}{2}, \frac{1}{s}+\frac{1}{2}\right)$, where $r=\sqrt{x^{2}+y^{2}}, G(r, a)=\frac{1}{\Gamma(a)} \int_{r}^{a} e^{-t} t^{a-1} d t$, and $\Gamma$ denotes the gamma-function. The parameters $\varepsilon$ (which will be negative, as we concentrate on depressions) and $s$ determine the amplitude and the steepness of the pressure anomaly, the maximum value of the pressure deviation $\Delta H / H$ depending on both. By stretching the zonal coordinate $x \rightarrow a^{-1} x$ in $H_{i}(x, y)$ we get a perturbation with the zonal to meridional aspect ratio $a$.

Numerical simulations were performed with high-resolution well-balanced finite-volume scheme [13] in a rectangular domain with sponges at the boundaries. The sponges allow to mostly evacuate short inertia-gravity waves, which are produced at initial stages of adjustment. No explicit dissipation was added and a second-order scheme [13] was used. The domain is symmetric with respect to the equator and wide enough, in order to minimize the influence of the boundaries: $28 L_{d} \times 28 L_{d}$, where $L_{d}=(g H)^{1 / 4} / \beta^{1 / 2}$ is the equatorial deformation radius. Maximal spatial resolution was $1200 \times 1200$ in the dry simulations, and $1400 \times 1400$ in MC simulations. The natural units of length and time are $L_{d}$ and $1 / \beta L_{d}$, respectively. Their numerical values depend on the interpretation of $g$ (full, or reduced gravity) and $H$, the non-perturbed thickness (full, or equivalent depth), e.g. for $g=9.8 \mathrm{~m} / \mathrm{s}^{2}, H \approx 10 \mathrm{~km}, L_{d} \approx 3000 \mathrm{~km}$ and $1 / \beta L_{d} \approx 5 h$. As usual in test cases [10], we start the MC simulations with a uniform initial moisture distribution $Q_{i}$ close to saturation: $Q_{i}=Q^{s}-0.01$ with $Q^{s}=0.9$, a short relaxation time $\tau$ equal to several time-steps of the numerical scheme, $\gamma=1$, and $\alpha=\mathscr{O}\left(10^{-1}\right)$.

The main result of experiments on the "dry" adjustment is a discovery of a significant difference between the evolution of pressure anomalies with large and order-one aspect ratios. A comparison of adjustments of circular and elongated negative pressure anomalies with $s=4$ and $\Delta H / H=$ 0.1 , as seen in relative vorticity and velocity fields, is presented in Fig. 1. As follows from the left column, the adjustment of a circular pressure anomaly in the eastern sector is similar to the standard Gill-like scenario, cf. [8], and is dominated by a Kelvin wave (KW), with characteristic velocity pattern although a signal corresponding to the eastward-propagating inertia-gravity wave $(\mathrm{EIGW})$ is also visible. However, it is totally different in the western sector, where a typical signal 

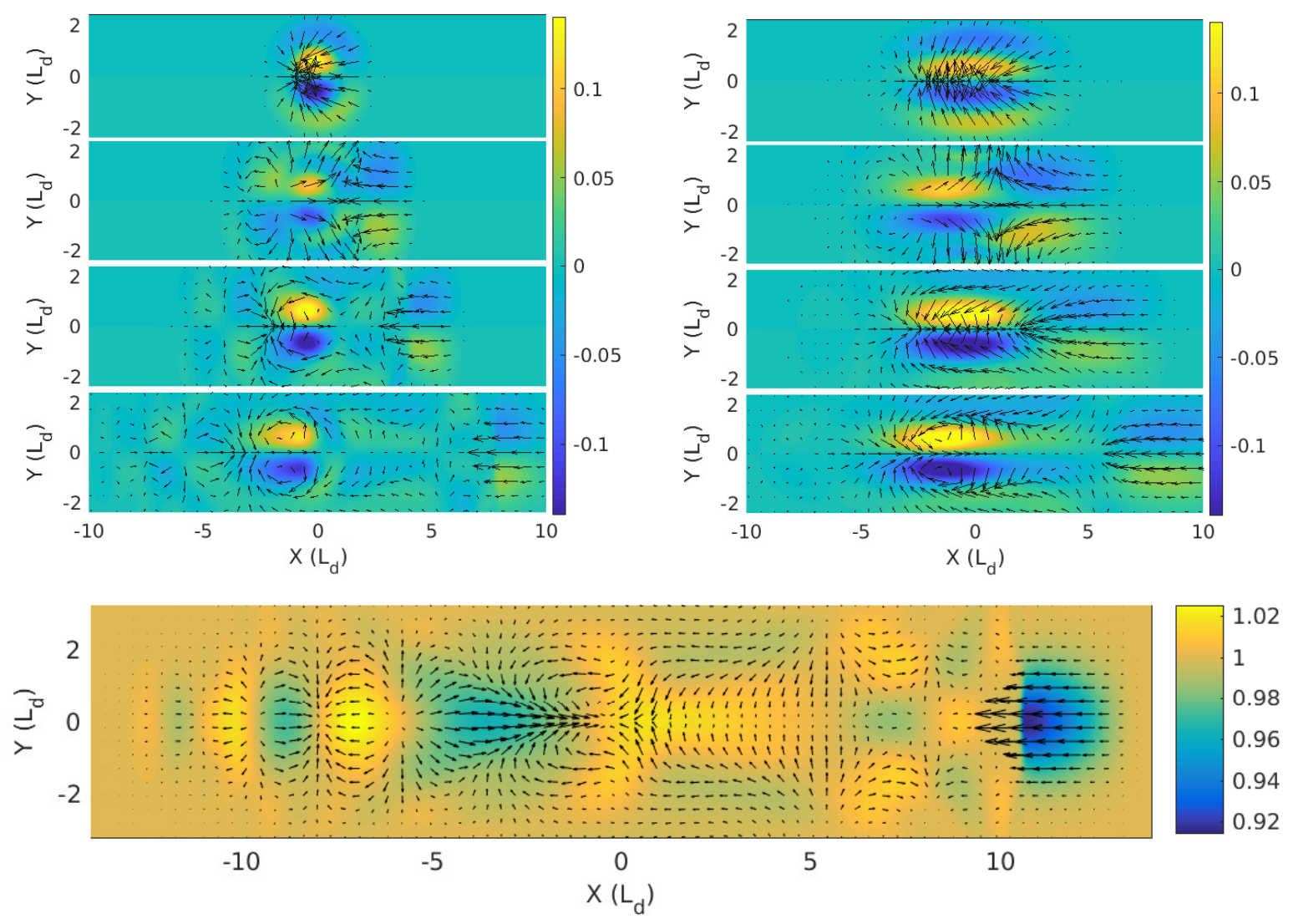

FIG. 1. Upper left panel: "Dry" adjustment of a circular pressure anomaly. Relative vorticity (colours), and velocity (arrows): $t=0,1,3,5,9\left[1 / \beta L_{d}\right]$ from top to bottom. Upper right panel: Same as in the left panel, but for a zonally elongated initial anomaly with aspect ratio $a=2.5$. Bottom panel: Snapshot of adjustment of the circular anomaly at a later time $t=12\left[1 / \beta L_{d}\right]$.

corresponding to the faster westward-propagating inertia-gravity wave (WIGW) with the structure of lowest meridional mode is clearly seen in the velocity field, in front of a slower propagating Rossby wave (RW) signal. This signal is even clearer identified at a later time $t=12\left[1 / \beta L_{d}\right]$, as follows from the lowermost panel and also Fig. 2. In comparison, the evolution of the elongated pressure anomaly is closer to the standard scenario with a well-pronounced RW structure, and less pronounced, although still present, WIGW structure (right panels). At large aspect ratios $a \geq 5$ the standard scenario of adjustment described in [8] is reproduced. The evolution of pressure (thickness) confirms these conclusions (not shown). The velocity patterns of lowest meridional modes of all equatorial waves (KW, RW, EIGW and WIGW), which are used for identifications, can be found e.g. in Zeitlin [7], Sect. 4.4. 

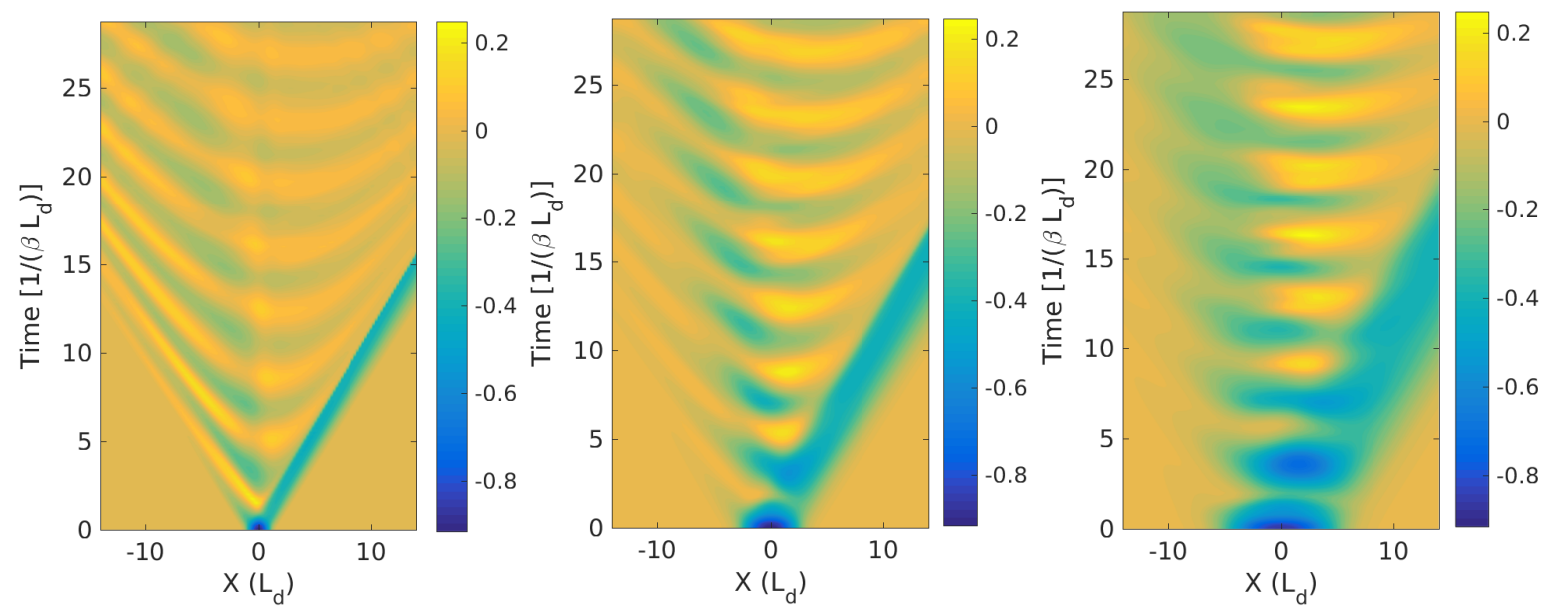

FIG. 2. Hovmöller diagrams of pressure signal along $y=1\left[L_{d}\right]$ corresponding, respectively, to the left and the right upper panels of Fig. 1 (left and middle panels), and to the adjustment of the pressure anomaly with large aspect ratio $a=5$ (right panel) .

The main result of the numerical simulations of the MC adjustment is a discovery of new scenarios with generation of steady eastward-moving twin cyclones, or cyclones moving, first, eastward, then westward, and detaching from the Equator. Smaller-scale intense vortices moving off the Equator also appear. In MC case we used exactly the same initial pressure perturbations as in the dry case. We found that, although both circular and zonally elongated weak initial anomalies produce a Gill - like scenario, cf. Fig. 3, the MC adjustment of the circular anomaly in the westward sector clearly generates WIGW. What is most important, when the amplitude of the anomaly increases at the same aspect ratio, the scenario of MC adjustment drastically changes, as follows from Fig. 4. While the initial perturbation still produces a Kelvin wave in the eastern sector, and inertia-gravity waves in the western sector, a new structure emerges: an intense twin dipole. The dipole arises from the vorticity pattern appearing at the early stages (cf. upper panel of Fig. 5 ), which becomes a pair of cyclones starting eastward and ejecting vorticity filaments, as follows from the top panels in Fig. 5. The fate of this dipole depends on the amplitude of the initial perturbation. For circular perturbations with $0.15<\max |\Delta H / H|<0.175$ the dipole stops moving eastward after some time, and engages in a reverse motion. Its northern and southern components start moving off the Equator in North-West and South-West directions, respectively, merging on their way with the smaller vortices, previously formed by the vortex filaments, and thus intensifying. For circular perturbations with $\max |\Delta H / H|>0.175$, the dipole ejects the filaments, which 

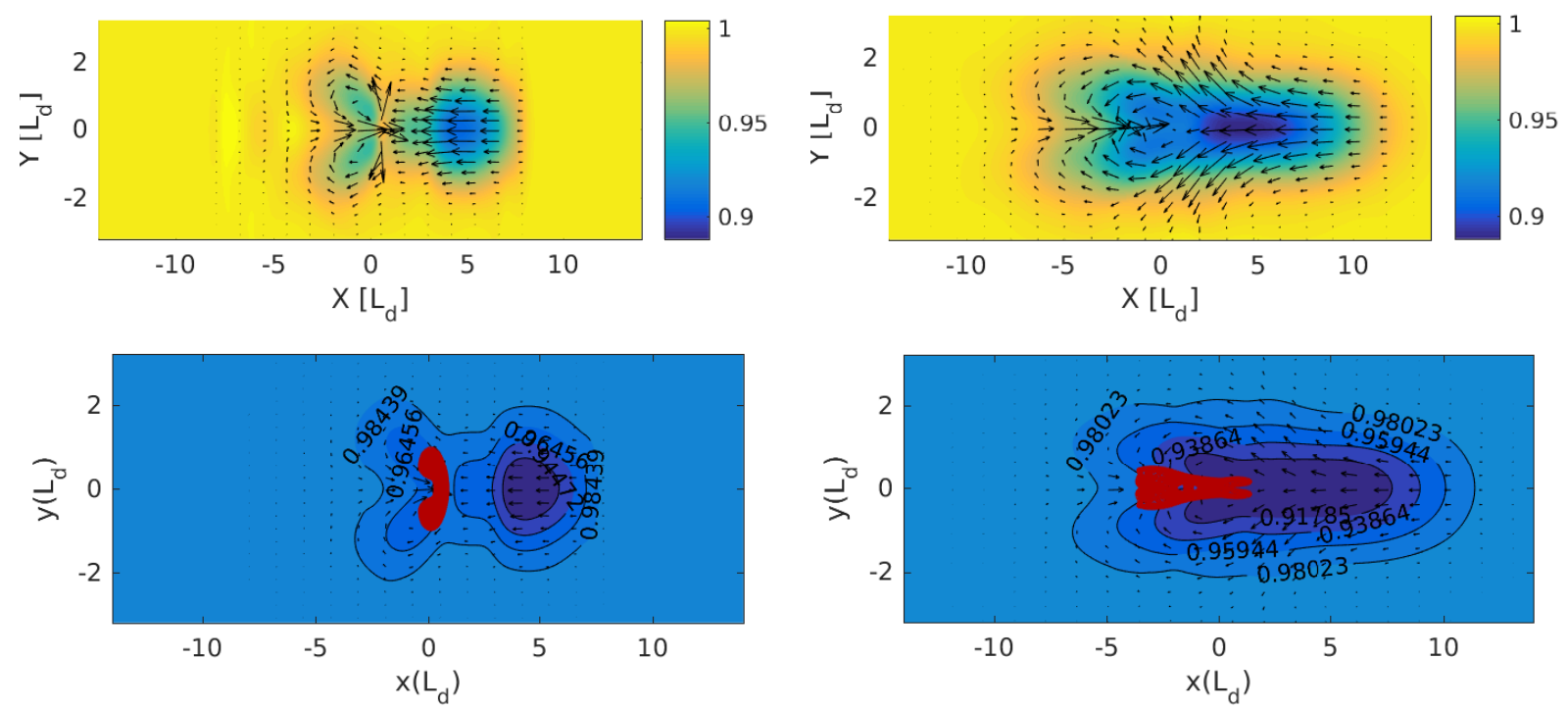

FIG. 3. From top to bottom: Snapshots at $t=7\left[1 / \beta L_{d}\right]$ of pressure (colours) and velocity (arrows), and pressure (contours) and condensation (red), during adjustment of negative pressure anomalies with $\max |\Delta H / H|=0.1$, and $a=1$ (left column) and $a=5$ (right column).
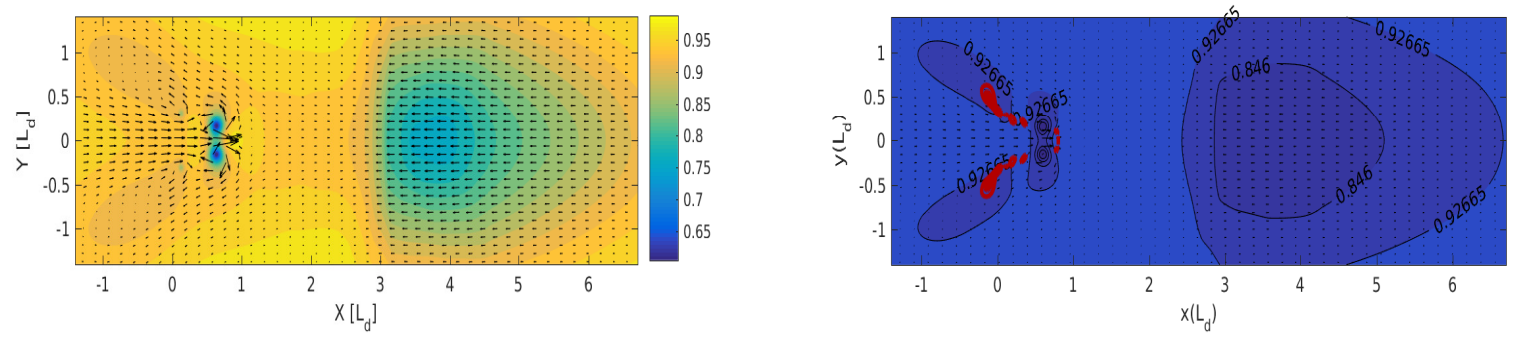

FIG. 4. Snapshots at $t=7\left[1 / \beta L_{d}\right]$ of pressure (colours) and velocity (arrows) (left panel), and pressure (contours) and condensation (red) (right panel) during MC adjustment of a circular negative pressure anomaly with $\max |\Delta H / H|=0.15$.

form secondary small-scale intense vortices left behind and moving westward and off the Equator, and continue a steady eastward motion. Both scenarios are presented in Fig. 5. The cyclonic dipole emerging from sufficiently strong anomalies keeps moving slowly eastwards, and is accompanied by a persistent condensation pattern (not shown). The structure of such dipoles strongly resembles that of the moist-convective eastward-moving equatorial modons recently described in [14].

Recently the equatorial modons were proposed as a possible dynamical explanation of the MJO in the dry shallow-water model in spherical geometry [15] and in mcRSW on the equatorial 

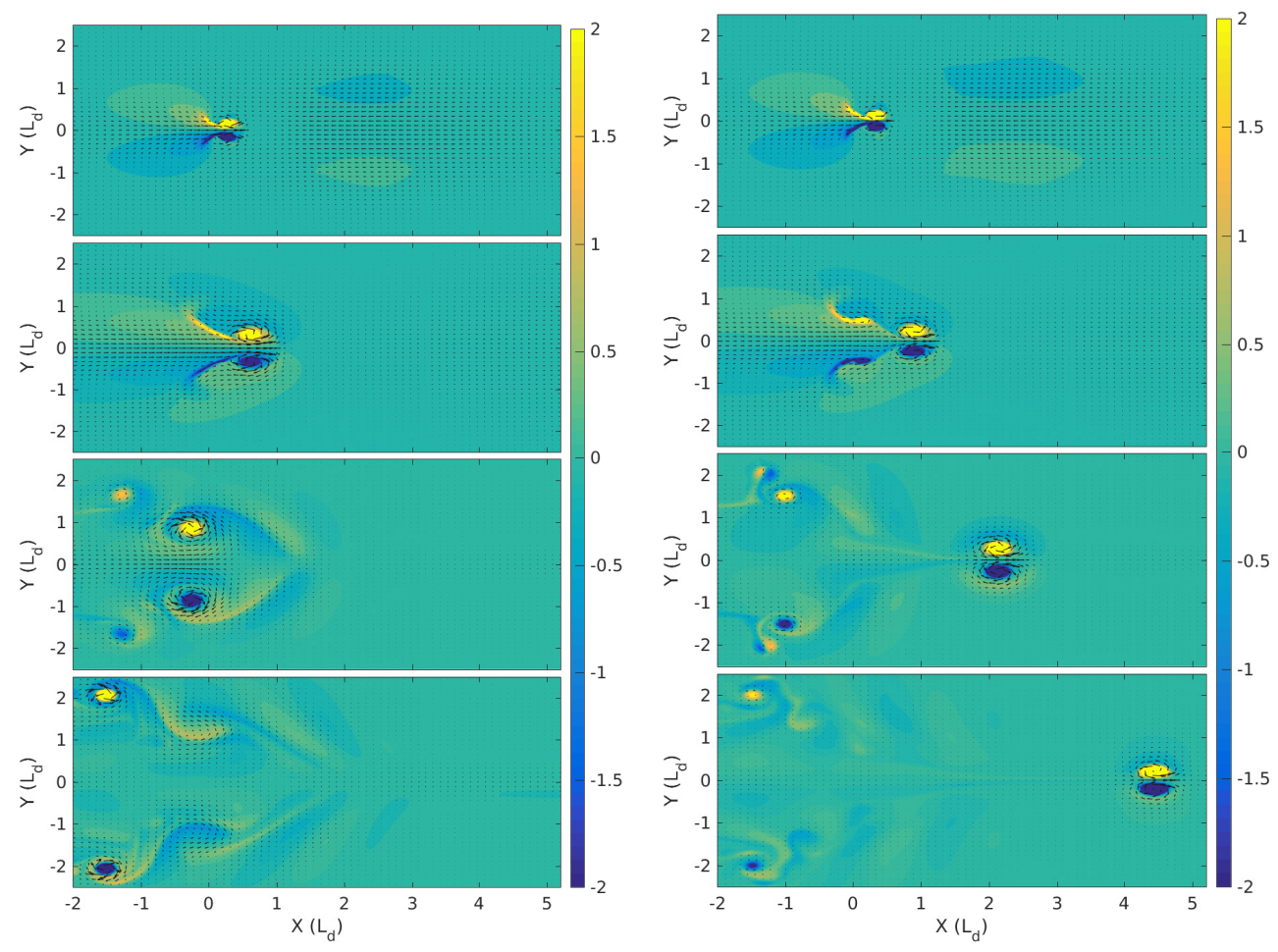

FIG. 5. Evolution at later times $t=5,30,45,75\left[1 / \beta L_{d}\right]$ of relative vorticity during the MC adjustment of circular negative pressure anomalies with $\max |\Delta H / H|=0.15$ (left panel), and $\max |\Delta H / H|=0.18$ (right panel)

beta-plane [14]. We hereby exhibit a mechanism of generation of such structures from pressure anomalies. We should stress that without the effects of moisture even strong depressions, e.g. with $\Delta H / H=0.25$, do not produce modons. The difference between initial stages of dry and moistconvective adjustments with $\Delta H / H=0.1$, and the role of convergence due to moist convection, are clearly seen in Fig. 6. At the very beginning, an almost isotropic inflow starts toward the center of the depression. Gradually the direction of the winds deviate from the center because of the Coriolis force. From this step on "dry" and MC adjustment follow two different evolution scenarios. While the "moon like" vorticity distributions become intensified, localized, and compactified because of moist-convection, it is not the case in "dry" adjustment. Once the emerging dipole acquires a vorticity large enough, it propagates eastward as an equatorial modon, otherwise other aforementioned scenarios are realized. The transient moist-convective scenario, when the eastward-moving dipole transforms into a pair of cyclones moving off the Equator can be of interest in the context of the genesis of tropical cyclones, and their possible link to Madden-Julian 

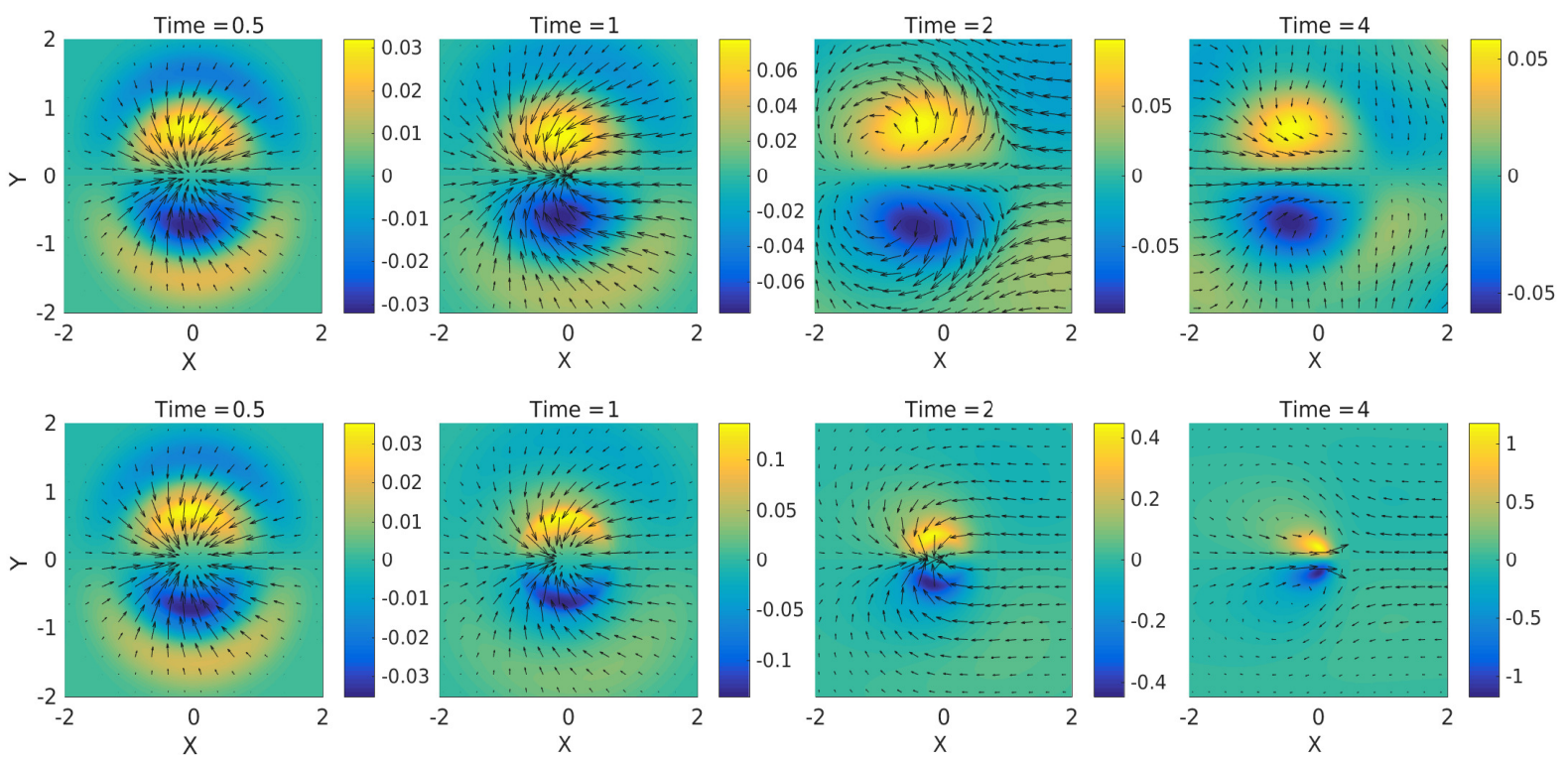

FIG. 6. Snapshots of vorticity (colors) and velocity (arrows) of the initial stages of "dry" (top) and MC (bottom) adjustments of a circular negative pressure anomaly with $\max |\Delta H / H|=0.1$. Notice a pronounced convergence at $t=2\left[1 / \beta L_{d}\right]$ due to condensation in the bottom panel.

events. In the study [3] this link was investigated using the "dry" RSW model on the sphere where the MJO was modeled as a stationary, or slowly eastward-moving, meridionally elongated mass sink acting for several days. A scenario close to our results for initial anomaly of intermediate intensity, cf. Fig. 5, left panel, was observed with a stationary sink of finite duration, while eastward-moving sink was producing a scenario resembling that of the right panel of Fig. 5. We displayed results for negative initial pressure perturbations symmetric with respect to the equator. The positive ones do not produce eastward-moving dipoles in the MC case, while in the dry case the results are similar. Relaxing the symmetry with respect to Equator would lead to appearance of Yanai waves in the response of the system to initial perturbations.

This work was supported by the French National program LEFE-IDAO. 
[1] A. Gill, "Some simple solutions for heat induced tropical circulation," Q. J. Roy. Met. Soc. 106, $447-$ $462(1980)$.

[2] W.A. Heckley and A. Gill, "Some simple analytical solutions to the problem of forced equatorial long waves,” Q. J. Roy. Met. Soc. 110, 203-217 (1984).

[3] R. Nieto Ferreira, W.H. Schubert, and J.J. Hack, "Dynamical aspects of twin tropical cyclones associated with the madden-julian oscillation,” J. Atmos. Sci. 53, 929-945 (1996).

[4] A. Gill, Atmosphere - Ocean Dynamics (Academic Press, 1982).

[5] T. Matsuno, "Quasi-geostrophic motions in the equatorial area," J. Met. Soc. Japan 44, 25-43 (1966).

[6] G.N. Vallis, Atmospheric and Oceanic Fluid Dynamics (Cambridge University Press, 2017) p. 314.

[7] V. Zeitlin, Geophysical Fluid Dynamics: Understanding (almost) everything with rotating shallow water models (Oxford University Press, 2018).

[8] J. LeSommer, G.M. Reznik, and V. Zeitlin, "Nonlinear geostrophic adjustment of long-wave disturbances in the shallow-water model on the equatorial beta-plane,” J. Fluid Mech. 515, 135-170 (2004).

[9] Y. Matsuda and H. Takayama, "Evolution of disturbances and geostrophic adjustment on the sphere," J. Met. Soc. Japan 67, 949-965 (1989).

[10] F. Bouchut, J. Lambaerts, G. Lapeyre, and V. Zeitlin, "Fronts and nonlinear waves in a simplified shallow-water model of the atmosphere with moisture and convection,” Phys. Fluids 21, 116604 (2009).

[11] M. Rostami and V. Zeitlin, "Improved moist-convective rotating shallow water model and its application to instabilities of hurricane-like vortices,” Q. J. Roy. Met. Soc. 144, 1450-1462 (2018).

[12] M. Rostami and V. Zeitlin, "Influence of condensation and latent heat release upon barotropic and baroclinic instabilities of atmospheric vortices in a rotating shallow water model on the f-plane," Geoph. Astrophys. Fluid Dyn. 111, 1-31 (2017).

[13] F. Bouchut, "Chapter 4: Efficient numerical finite volume schemes for shallow water models," in Nonlinear Dynamics of Rotating Shallow Water: Methods and Advances, Edited Series on Advances in Nonlinear Science and Complexity, Vol. 2, edited by V. Zeitlin (Elsevier, 2007) pp. 189 - 256.

[14] M. Rostami and V. Zeitlin, "Eastward-moving convection-enhanced modons in shallow water in the equatorial tangent plane,” Phys. Fluids 31, 021701 (2019).

[15] J.-I. Yano and J.J. Tribbia, “Tropical atmospheric Madden-Julian oscillation: a strongly nonlinear free 
solitary Rossby wave?” J. Atmos. Sci. 74, 3473-3489 (2017). 